\title{
Calcitonin gene-related peptide induces IL-6 expression in RAW264.7 macrophages mediated by mmu_circRNA_007893
}

\author{
TIAN DENG, LAN YANG, ZHICHAO ZHENG, YUANJING LI, WEN REN, CAIJUAN WU and LVHUA GUO
}

\author{
Key Laboratory of Oral Medicine, Guangzhou Institute of Oral Disease, Stomatology Hospital \\ of Guangzhou Medical University, Guangzhou, Guangdong 510140, P.R. China
}

Received December 29, 2016; Accepted August 14, 2017

DOI: $10.3892 / \mathrm{mmr} .2017 .7779$

\begin{abstract}
Several circular RNAs (circRNAs) may have role important roles in biological processes, however, there is limited knowledge of circRNAs and their potential functions in RAW264.7 macrophages. The present study aimed to examine the expression of circRNAs and explore their effects on interleukin-6 (IL-6) expression induced by calcitonin gene-related peptide (CGRP) in RAW264.7 macrophages. To identify circRNAs, the circRNA expression was measured in macrophages with or without CGRP stimulation. The interaction between circRNAs and microRNAs (miRs) were then identified using bioinformatic software and networks. In the current study, it was demonstrated that CGRP increased the expression of IL- 6 mRNA in a dose- and time-dependent manner. Furthermore, mmu_circRNA_007893 was significantly increased in the CGRP-stimulated macrophages. Silencing of mmu_circRNA_007893, IL-6 mRNA expression was significantly decreased, whereas mmu-miR-485-5p expression was markedly increased. Furthermore, when overexpression of mmu-miR-485-5p, IL-6 mRNA was markedly decreased. The results demonstrated that CGRP-induced IL-6 mRNA expression was mediated by mmu_circRNA_007893, and mmu_circRNA_007893 functioned as an endogenous mmu-miR-485-5p sponge as part of induction of IL-6 mRNA expression.
\end{abstract}

\section{Introduction}

Calcitonin gene-related peptide (CGRP) is a 37-amino acid peptide with a variety of biological effects, and is widely distributed in peripheral and central nervous system (1). Initially, it

Correspondence to: Dr Lvhua Guo or Dr Lan Yang, Key Laboratory of Oral Medicine, Guangzhou Institute of Oral Disease, Stomatology Hospital of Guangzhou Medical University, 39 Huangsha Road, Guangzhou, Guangdong 510140, P.R. China

E-mail: fsglh1@163.com

E-mail: yang11900@163.com

Key words: calcitonin gene-related peptide, circular RNA, interleukin-6, macrophages, microRNA was reported that CGRP is produced by sensory nerve cells and has effect on sympathetic nervous pathways (2). With the development of research, it has been confirmed that CGRP has an important role in the cardiovascular system, respiratory system and skeletal system (3). Furthermore, there is growing concern about the association between CGRP and the immune system. As one of the most important neurotransmitters of the neuroimmune axis, CGRP works as a bridge connecting the nervous system and immune system (4-6). Furthermore, the research on CGRP and macrophages has become a hot topic in the past decades. CGRP can induce macrophages to produce cytokines through interaction with CGRP receptors. The calcitonin receptor-like receptor and receptor activity-modifying protein 1 are present on the surface of bone marrow-derived macrophages (7). Additionally, $\alpha$-CGRP receptors are present on alveolar macrophages (8). CGRP has an important role on the inflammatory response mediated by lipopolysaccharide (LPS) in macrophages $(9,10)$ and CGRP is also be secreted by macrophages (11). It has been proved that certain classical pathways are involved in biological processes, including inflammatory response, the cyclic adenosine monophosphate pathway and nuclear factor- $\kappa B$ pathway (9-12).

Circular RNA (circRNA) is a special type of non-coding RNA that was first identified 20 years ago; however, it was not until recent years that the study of circRNA function and structure became common $(13,14)$. CircRNA is characterized by the presence of a covalent bond linking the $3^{\prime}$ and 5 'ends generated by back splicing (15). Although circRNA can be derived from introns, untranslated regions and gene fragments, it is predominantly produced from exonic DNA encodes protein $(15,16)$. The function of circRNA remains unclear; however, research has provided some information. CircRNA is widely expressed in human cells and the abundance of it exists in cytoplasm $(17,18)$. The sequences of the majority of circRNAs are highly conserved, and have the ability to mediate gene expression at the transcriptional level or post-transcriptional level by altering their downstream miRNAs expression $(15,18)$. Furthermore, circRNA is more stable than linear RNA in cells, as its unique structure is resistant to degradation by exonucleases (16). Recently, it was reported that circRNA can act as a 'sponge' for microRNAs (miRNAs) (19). The previous study demonstrated that cerebellar degeneration-related protein 1 transcript antisense RNA (CDR1as) acts as a circular miRNA-7 (miR-7) inhibitor, 
which harbors more than 70 conventional miR-7 binding sites. When CDR1 as is overexpressed, miR-7 will associate with it and the expression of miR-7 target genes increases (20); this is termed the 'sponge effect' or competing endogenous RNA mechanism, and this effect has been validated in multiple studies (21-23).

In the present study, it was demonstrated that interleukin-6 (IL-6) mRNA expression in RAW264.7 macrophages is enhanced by CGRP in a dose- and time-dependent manner. Furthermore, the expression of certain circRNAs, including mmu_circRNA_007893, was demonstrated to be changed when macrophages were stimulated by CGRP. Subsequently, the function and mechanism of mmu_circRNA_007893 was investigated. The results of the current study demonstrated that mmu_circRNA_007893 is involved in IL-6 mRNA induction by CGRP in RAW264.7 macrophages.

\section{Materials and methods}

Cell culture. The RAW264.7 macrophages (Infrastructure of Cell Line Resources, Beijing, China) were plated on 6-well plate culture dishes at density of $2 \times 10^{5}$ cells per well in $2 \mathrm{ml}$ of Dulbecco's modified Eagle's medium (Gibco; Thermo Fisher Scientific, Inc., Waltham, MA, USA) containing $10 \%$ fetal bovine serum (Gibco; Thermo Fisher Scientific, Inc.). The 6-well plates were placed at $37^{\circ} \mathrm{C}$ in a humidified atmosphere of $95 \%$ air and $5 \% \mathrm{CO}_{2}$. Media were replaced $1 \mathrm{~h}$ before the experiments.

Verification of optimum concentration and time point of CGRP on RAW264.7 cell lines. In the concentration experiments, the cells were divided into 6 groups: $0.01,0.1,1,10,100 \mathrm{nM}$ CGRP and the control group. Then cells were treated with CGRP (Sigma-Aldrich; Merck KGaA, Darmstadt, Germany) at the corresponding concentration. After stimulation for $2 \mathrm{~h}$, the total RNA was collected and the expression of IL-6 in each group was detected by reverse transcription-quantitative polymerase chain reaction (RT-qPCR). Similarly, in the time point experiments, the cells were divided into 5 groups: 4, 3 , $2,1 \mathrm{~h}$ and the control group $(0 \mathrm{~h})$. Then, cells were treated with $1 \mathrm{nM}$ CGRP for the corresponding time. The total RNA was collected and the expression of IL-6 in each group was detected by RT-qPCR.

Preparation and analysis of the circRNA microarrays. The cells were divided into 2 groups: Control group and subject group. In the subject group, the cells were treated with $1 \mathrm{nM}$ CGRP for $2 \mathrm{~h}$. In the control, the cells were untreated. The total RNA of the 2 groups was isolated using TRIpure (Aidalb Biotechnologies Co., Ltd., Beijing, China) and RNA in each group was quantified using NanoDrop 2000 (Thermo Fisher Scientific, Inc., Wilmington, DE, USA). The samples were prepared and hybridized based on the Arraystar standard protocols (Arraystar, Inc., Rockville, MD, USA). In brief, according to the Arraystar Super RNA Labeling protocol (Arraystar, Inc.), total RNA of samples was amplified and transcribed into fluorescent cRNA utilizing random primers. The labeled cRNAs were hybridized onto the Arraystar Human circRNA Array (8x15K, Arraystar, Inc.). After washing the slides, the arrays were scanned with an Agilent G2505C Scanner. Then,
Agilent Feature Extraction software (version 11.0.1.1) was used to analyze the acquired array images. Quantile normalization and subsequent data processing were performed with the R software package. After the circRNA microarray was completed, all the circRNAs that had changed above 2 folds were displayed in the circRNA profiling data (The data was provided by the Arraystar, Inc.). Then, the circRNAs were selected with reference to the networks using circBase (http://www. circbase.org), mirBase (http://www.mirbase.org) and miRDB (http://www.mirdb.org); the selected circRNA were confirmed by RT-PCR and their PCR products were sequenced (Nuclea Bio-tech Inc., Guangzhou, China).

Fluorescence in situ hybridization (FISH). The probe used to detect mmu_circRNA_007893 was synthesized by BersinBio (Guangzhou, China) and its sequence was CTCCAGCTT GCCTTCATCCAGTCCAA. The FISH experiment was performed with the BersinBio ${ }^{\mathrm{TM}}$ circRNA FISH kit. The cells were fixed by $4 \%$ paraformaldehyde at room temperature for $20 \mathrm{~min}$ and washed with diethyl pyrocarbonate twice. Prior to permeabilization, the cells were washed by $1 \mathrm{X}$ PBS and fixed by $4 \%$ paraformaldehyde at room temperature for $10 \mathrm{~min}$. Then the samples were washed by $1 \mathrm{X}$ PBS three times and permeabilized at $4^{\circ} \mathrm{C}$ for $5 \mathrm{~min}$. The probe labeled with fluorescein isothiocyanate (100 $\mathrm{ng} / \mu \mathrm{l}$; BersinBio) and hybridization solution (BersinBio) were mixed at the ratio of 1:19 and denaturated at $73^{\circ} \mathrm{C}$ for $8 \mathrm{~min}$. Then, the mixture was added to the samples and the samples were incubated overnight at $42^{\circ} \mathrm{C}$. In the present study, DAPI $(5 \mu \mathrm{g} / \mathrm{ml}$; Beyotime Institute of Biotechnology, Shanghai, China) was used to stain the nucleus at room temperature for $10 \mathrm{~min}$. The images were observed with a confocal microscope (Leica Microsystems $\mathrm{GmbH}$, Wetzlar, Germany).

circRNA interference. The small interfering RNAs (siRNAs) targeting mmu_circRNA_007893 were designed and synthesized by Guangzhou RiboBio Co., Ltd. (Guangzhou, China). The sequences of siRNA1-3 were showed as followed: siRNA1, 5'-GCCUUCAUCCAGUCCAAUGdTdT-3' (sense) and 3'-dTdTCGGAAGUAGGUCAGGUUAC-5' (antisense); siRNA2, 5'-CAGCUUGCCUUCAUCCAGUdTdT-3' (sense), 3'-dTdTGUCGAACGGAAGUAGGUCA-5' (antisense); and siRNA3, 5'-CCCUCCAGCUUGCCUUCAUdTdT-3' (sense) and 3'-dTdTGGGAGGUCGAACGGAAGUA-5' (antisense), respectively. Negative siRNA was used as a control. The RAW264.7 macrophages were transfected with siRNA using GenMute $^{\mathrm{TM}}$ siRNA Transfection Kit and Transfection Buffer (SignaGen Laboratories, Rockville, MD, USA) according to the manufacturer's instructions. Then the cells were incubated for $48 \mathrm{~h}$ at $37^{\circ} \mathrm{C}$ prior to the sequential tests.

miRNA overexpression and inhibition. The mmu-miR-485-5p mimic and inhibitor were synthesized by Invitrogen (Thermo Fisher Scientific, Inc.). The sequences were as follows: Mimic, 5'-AGAGGCUGGCCGUGAUGAAUUC-3' (sense) and 3'-UCUCCGACCGGCACUACUUAAG-5' (antisense); inhibitor, 5'-UCUCCGACCGGCACUACUUAAG-3'. The culture media were replaced $1 \mathrm{~h}$ before transfection. Then the cells were transfected with mimics or inhibitor using Lipofectamine 3000 (Invitrogen; Thermo Fisher Scientific, Inc.) following the 
A

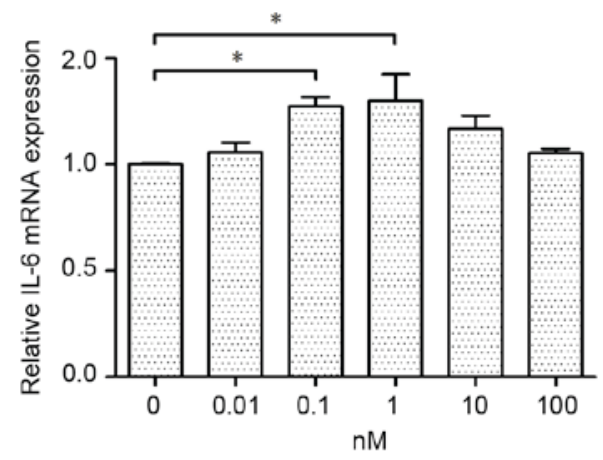

B

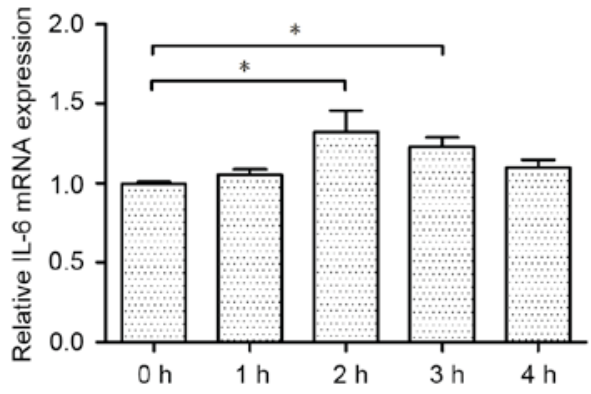

Figure 1. CGRP induced changes to the IL- 6 mRNA levels in a dose- and time-dependent manner. (A) Cells were treated for up to $2 \mathrm{~h}$ with CGRP at concentration ranging from 0.01 to $100 \mathrm{nM}$. The optimum concentration was $1 \mathrm{nM}$ and the IL-6 mRNA expression in following treatment with $0.1 \mathrm{nM}$ ( $\mathrm{P}=0.019)$ and $1 \mathrm{nM}(\mathrm{P}=0.011)$ were significantly higher than that in the $0 \mathrm{nM}$ group. (B) Cells were treated with CGRP at concentration of $1 \mathrm{nM}$ for $1-4 \mathrm{~h}$. The optimum treatment duration was $2 \mathrm{~h}$ and the IL- 6 mRNA expression at $2 \mathrm{~h}(\mathrm{P}=0.011)$ and $3 \mathrm{~h}(\mathrm{P}=0.044)$ was significantly higher than that in the $0 \mathrm{~h}$ group. The IL-6 mRNA was detected by reverse transcription-quantitative polymerase chain reaction. The values are presented as the mean \pm standard error. ${ }^{*} \mathrm{P}<0.05, \mathrm{n}=3$ for each group. CGRP, calcitonin gene-related peptide; IL-6, interleukin-6.

manufacturer's protocol and incubated for $24 \mathrm{~h}$ at $37^{\circ} \mathrm{C}$ prior to the subsequent experiments.

Detection of IL-6 mRNA, mmu_circRNA_007893, $m m u-m i R-485-5 p$ by $R T-q P C R$. Total RNA from RAW264.7 cell line was extracted using TRIzol reagent (Thermo Fisher Scientific, Inc.). The mRNA samples were treated with DNase to eliminate contaminating genomic DNA and reverse transcribed using the First Strand cDNA Synthesis Kit (Takara Bio, Inc., Otsu, Japan). For circRNA analysis, total RNA was reverse transcribed using random primers (Invitrogen; Thermo Fisher Scientific, Inc.). miRNAs were extracted using small RNAiso (Takara Bio, Inc.) and reverse transcribed by MicroRNA First-Strand Synthesis Kits (Takara Bio, Inc.). GAPDH was chosen to be the internal standard for mRNA and circRNAs analysis and U6 was selected to the internal standard for miRNA analysis. The primers used in the experiment as follows: IL-6, 5'-CCACTTCACAAGTCGGAGGCT TA-3' (forward) and 5'-CCAGTTTGGTAGCATCCATCA TTTC-3' (reverse); mmu_circRNA_007893, 5'-CAGTAA GGGAGGGCAAGAA-3' (forward) and 5'-GCCATAAGG TGGAATCTGC-3' (reverse); mmu-miR-485-5p, 5'-GCT GGCCGTGATGAATTCAAA-3' (forward); and GAPDH, 5'-AAGAAGGTGGTGAAGCAGG-3' (forward) and 5'-GAA GGTGGAAGAGTGGGAGT-3' (reverse). The RT-qPCR was undertaken with the SYBR ${ }^{\circledR}$ Premix Ex Taq ${ }^{\mathrm{TM}}$ II kit (Takara Bio, Inc., Otsu, Japan). Briefly, the total reaction system was $25 \mu \mathrm{l}$ and the RT-qPCR was performed using 40 cycles with a $10 \mathrm{~min}$ denaturation at $95^{\circ} \mathrm{C}, 15 \mathrm{sec}$ annealing at $95^{\circ} \mathrm{C}$ and a 1 min extension at $60^{\circ} \mathrm{C}$. The $2^{-\Delta \Delta \mathrm{Cq}}$ method was used to analyze the results (24).

Western blotting. Protein was extracted using radioimmunoprecipitation assay buffer (50 mM Tris $\mathrm{HCl}, \mathrm{pH} 7.4,150 \mathrm{mM}$ $\mathrm{NaCl}, 1 \%$ Nonidet $\mathrm{P} 40$ and $0.1 \%$ sodium dodecyl sulfate) and phenylmethanesulfonyl fluoride at $4^{\circ} \mathrm{C}$. Bicinchoninic acid protein assay kit (BestBio, Shanghai, China) was used to determine the concentration. The concentration of each sample was $500 \mathrm{ng} / \mu \mathrm{l}$ and all the samples were run on $10 \%$ sodium dodecyl sulfate-polyacrylamide gel and electro-transferred onto polyvinylidene fluoride membranes for $1 \mathrm{~h}$ at $4^{\circ} \mathrm{C}$. After being electro-transferred, the polyvinylidene fluoride membranes were blocked by skimmed milk for $1 \mathrm{~h}$ at room temperature. Antibodies against GAPDH (97166; 1:4,000; Cell Signaling Technology, Inc., Danvers, MA, USA) and IL-6 (sc-57315; 1:200; Santa Cruz Biotechnology, Inc., Dallas, TX, USA) were incubated with blots overnight at $4{ }^{\circ} \mathrm{C}$. Then the blots were incubated with secondary antibody (anti-mouse IgG; ab6789; 1:5,000; Abcam, Cambridge, $\mathrm{UK}$ ) at room temperature for $1 \mathrm{~h}$. Proteins were detected by electrochemiluminescence (Beijing Dingguo Changsheng Biotechnology Co., Ltd., Beijing, China).

Statistical analysis. The SPSS version 20.0 program (IBM Corp., Armonk, NY, USA) was used for the statistical analysis. The data of two groups were analyzed by the Student's t test. The data of three or more groups were evaluated using analysis of variance and least significant difference post hoc tests when the variance was normal, otherwise Dunnett's T3 test was used. The results are reported as the mean \pm standard error. $\mathrm{P}<0.05$ was considered to indicate a statistically significant difference.

\section{Results}

CGRP induces IL-6 mRNA expression in RAW264.7 macrophages. To identify the effect of CGRP on IL- 6 mRNA expression in RAW264.7 macrophages, cultured cells were treated for different times, with different concentrations of CGRP. IL-6 mRNA was measured by RT-qPCR. In the concentration experiments, macrophages were stimulated with CGRP (0.01 to $100 \mathrm{nM}$ ) for up to $2 \mathrm{~h}$. As presented in Fig. 1A, the promotion effect of CGRP was observed at a concentration of $10 \mathrm{nM}$ and peaked at the concentration of $1 \mathrm{nM}$. The transient increase effect was not different to the basal level at a concentration of $0.01 \mathrm{nM}$. Therefore, $1 \mathrm{nM}$ CGRP was selected as the optimum concentration for the subsequent experiments. Similarly, CGRP caused a time-dependent change to IL-6 mRNA expression in RAW264.7 macrophages. The macrophages were treated with $1 \mathrm{nM}$ CGRP for 1-4 h. As presented in Fig. 1B, the expression of IL-6 mRNA increased gradually from 1 to $2 \mathrm{~h}$, and remained stable for $1 \mathrm{~h}$. Then, 


\begin{tabular}{lccc} 
A & & & \\
\hline Rank & circRNA & Fold change & Maximum predicted miRNA biding sites \\
\hline 1 & mmu_circRNA_016242 & 45.19 & 2 \\
2 & mmu_circRNA_013499 & 36.62 & 2 \\
3 & mmu_circRNA_015506 & 31.38 & 2 \\
4 & mmu_circRNA_005300 & 28.83 & 2 \\
\hline
\end{tabular}

B

\begin{tabular}{cccc}
\hline Rank & circRNA & Fold change & Maximum predicted miRNA biding sites \\
\hline 1 & mmu_circRNA_007893 & 2.35 & 10 \\
2 & mmu_circRNA_000476 & 2.22 & 7 \\
3 & mmu_circRNA_003022 & 2.21 & 7 \\
4 & mmu_circRNA_014201 & 5.53 & 6 \\
\hline
\end{tabular}

C

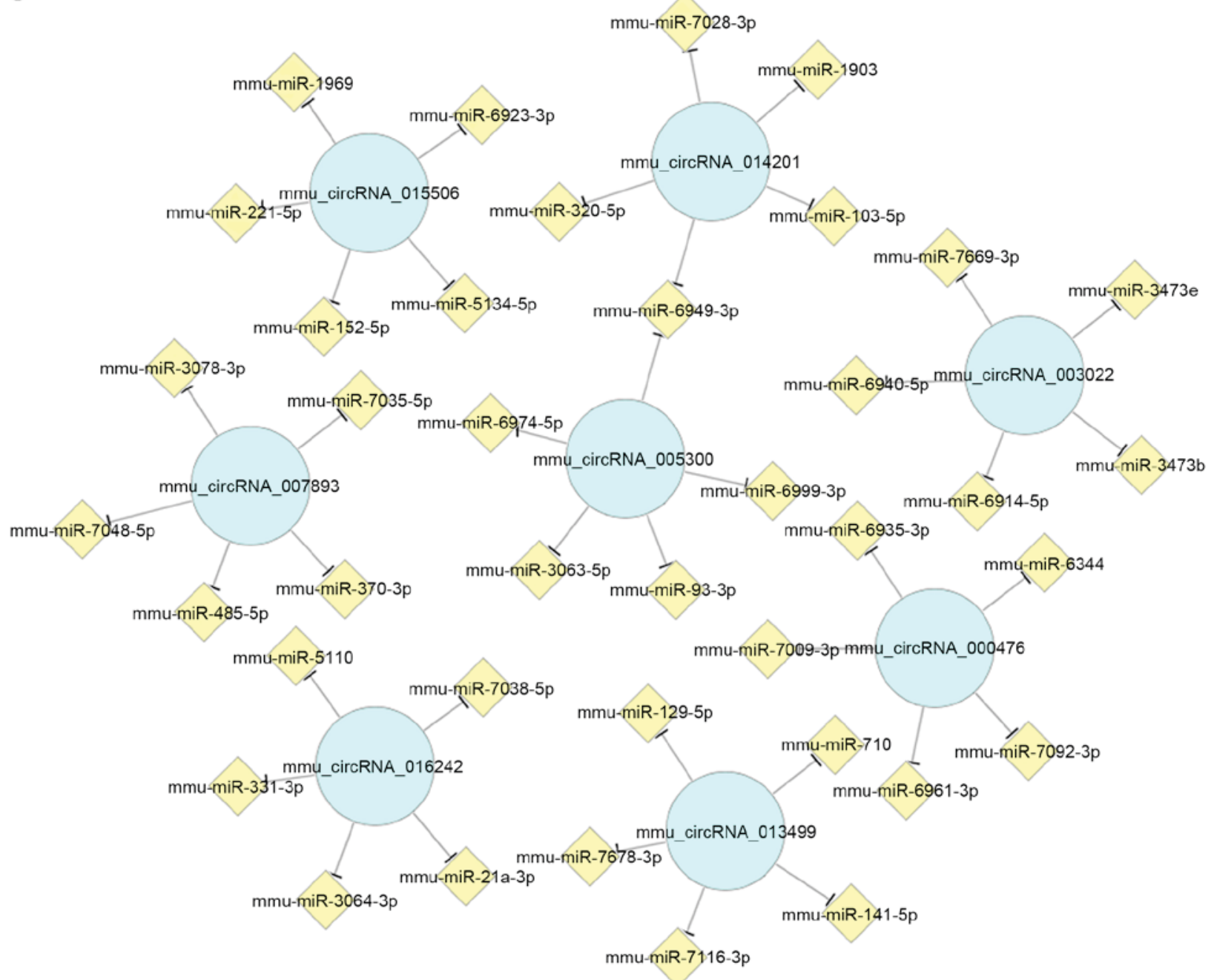

Figure 2. Upregulated circRNAs identified by in the circRNA microarray. (A) Top four upregulated circRNAs according to fold change. (B) Top four upregulated circRNA according to maximum miRNA binding sites. (C) Eight circRNAs and 24 predicted target miRNAs. In this network, the circle represents the circRNA and the diamond represents miRNAs. mmu, Mus musculus; circRNA, circular RNA; miRNA, microRNA. 
the IL-6 mRNA expression decreased slightly. These results suggested that CGRP stimulates dose- and time-dependent increases in IL-6 mRNA.

CGRP affects the expression of circRNAs in RAW264.7 macrophages. In the circRNA microarray, 349 circRNAs were changed $>2$-fold between the control group and subject group (1 nm CGRP for $2 \mathrm{~h}$ ). Among them, 173 circRNAs were significantly upregulated. The upregulated circRNAs were ranked according to the fold change and the top four are listed in Fig. 2A. CircRNAs can interact with their target miRNAs by base pairing and most have more than five miRNAs as potential targets. Furthermore, each miRNA can bind to several circRNAs. Thus, it is difficult to define which circRNA have the most important roles in biological processes. Therefore, it was assumed that the circRNAs that have more miRNA binding sites have more critical roles in the circRNA-miRNA network (23). According to this hypothesis, the upregulated circRNAs were ranked by the number of the predicted miRNA binding sites and the top four are listed in Fig. 2B. Furthermore, the target miRNAs of the former eight circRNAs were ranked according to mirSVR scores and the five highest ranking miRNAs for each circRNA are presented in Fig. 2C. Cytoscape was used to construct the circRNA-miRNA network.

CGRP promotes mmu_circRNA_007893 expression in $R A W 264.7$ macrophages. To confirm the validity of the data microarray, all the circRNAs identified were also analyzed by RT-qPCR. However, several circRNAs exhibited unchanged expression or the opposite trend compared with the microarray results. As presented in Fig. 3A, it demonstrated that mmu_circRNA_007893 expression increased stably by $\sim 2$ fold relative to the control group. The data was in accordance with the results in the circRNAs microarray. The result indicated that CGRP promotes mmu_circRNA_007893 expression in RAW264.7 macrophages. Furthermore, according to the circRNA microarray, mmu_circRNA_007893 were located mainly in the cytoplasm. To confirm the result, the fluorescent probes complementary to the junction sequences was attached to the circRNAs and fluorescence in situ hybridization (FISH) was used. As shown in Fig. 3B, the location of the circRNA was mostly in the cytoplasm.

CGRP promotes IL-6 expression by mediated mmu_ circRNA_007893 expression. Referring to the aforementioned results, CGRP induced mmu_circRNA_007893 and IL-6 mRNA expression in RAW264.7 macrophages. It was then explored whether IL-6 is a downstream mediator of mmu circRNA_007893. Three different siRNAs were used to reduce the circRNA expression. As demonstrated in Fig. 4A, all the siRNAs were effective and siRNA3 was selected as the optimum siRNA for the subsequent experiments. The IL-6 mRNA expression was detected following circRNA inhibition using the siRNA. In the CGRP + siRNA group, the cells were treated with siRNA3 for $48 \mathrm{~h}$ before CGRP stimulation. In Fig. 4B, IL-6 mRNA expression level declined correspondingly with the decrease of the circRNA. IL-6 proteins were also detected using western blotting. The result of western blot was consistent with that of RT-qPCR (Fig. 4C). Overall,
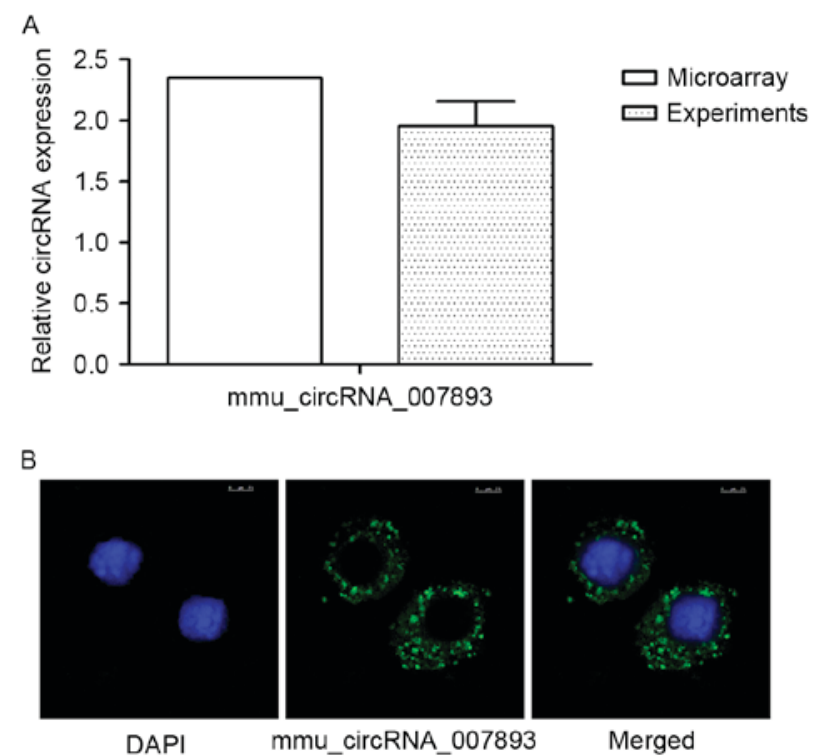

Figure 3. Validation of mmu_circRNA_007893 expression. (A) Fold change in expression of mmu_circRNA_007893 in RAW264.7 macrophages is determined by reverse transcription-quantitative polymerase chain reaction. The result was in accordance with the results of the microarray. Fold change in subject group normalized to the result of the microarray which showed no significant difference ( $\mathrm{P}=0.107 ; \mathrm{n}=4$ for each group). (B) Fluorescence in situ hybridization images of mmu_circRNA_007893 from the circRNA microarray, demonstrating the localization of circRNAs and nuclei of RAW264.7 macrophages (scale bar, $7.5 \mu \mathrm{m}$ ). circRNA, circular RNA; mmu, Mus musculus.

the data indicates that CGRP promotes IL-6 expression and mmu_circRNA_007893 is involved in this effect.

Role of mmu-miR-485-5p in mmu_circRNA_007893-mediated $I L-6$ release. It is reported that circRNAs may act as a 'sponge' for miRNAs, and to thus influence mRNA expression. Therefore, target miRNAs that may associate with circRNAs were identified using bioinformatic networks and the circRNA microarray. According to the circRNA microarrays, mmu-miR-485-5p was one of the predicated target miRNAs of mmu_circRNA_007893. As demonstrated in Fig. 5A, this mmu-miR-485-5p was predicted to match five regions of the complete sequence of mmu_circRNA_007893. To identify whether mmu-miR-485-5p took part in the biological processes in thus study, the mmu-miR-485-5p expression was determined in CGRP group and CGRP + siRNA group cells. Referring to Fig. 5B, the expression of miR-485-5p in the CGRP groups was significantly lower than that in the negative control groups. However, with mmu_circRNA_007893 expression knocked down by siRNA, the expression of miR-485-5p was rescued. This result suggests that mmu_circRNA_007893 reduces the expression level of mmu-miR-485-5p. Subsequently, whether IL-6 mRNA is downstream of mmu-miR-485-5p was investigated. miRNA mimics and inhibitor were transfected into cells and termed the CGRP + mimics and CGRP + inhibitor groups, respectively, for $24 \mathrm{~h}$ prior to CGRP stimulation. Transfection with mimics resulted in the enforced expression of mmu-miR-485-5p compared with the negative control, while the cells transfected inhibitors had decreased miR-485-5p levels, as analyzed by RT-qPCR (data not shown). As presented in Fig. 5C, IL-6 mRNA expression in the CGRP + mimics 
A

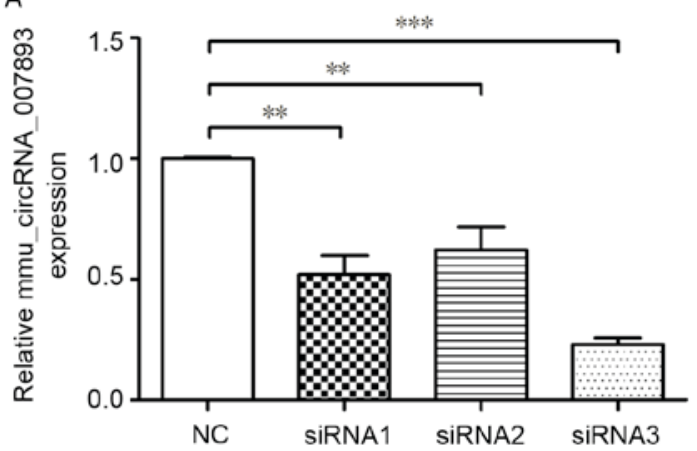

B

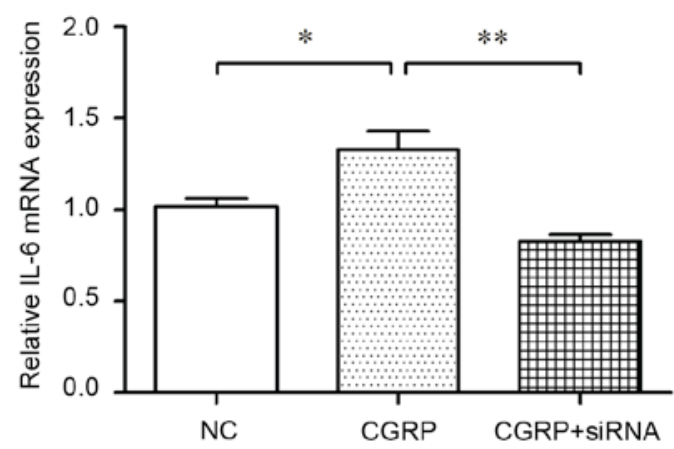

C

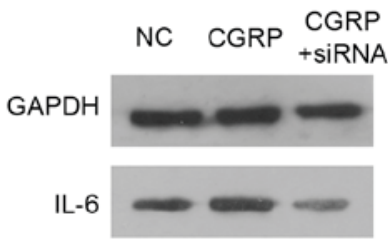

Figure 4. Effects of mmu_circRNA_007893 inhibition on IL-6 mRNA expression in RAW264.7 macrophages. (A) Cells were treated with three siRNAs targeting mmu_circRNA_007893. circRNA expressions in siRNA1 groups $(\mathrm{P}=0.001)$, siRNA2 groups $(\mathrm{P}=0.003)$ and siRNA3 groups $(\mathrm{P}<0.001)$ were significantly lower than that in negative control groups. (B) IL-6 mRNA expression in the CGRP-treated group was significantly higher than that in negative control group $(\mathrm{P}=0.018)$. However, its expression level in CGRP + siRNA groups decreased following knockdown of mmu_circRNA_007893 using siRNA3, which was markedly lower than that in the CGRP groups $(\mathrm{P}=0.002)$. The IL- 6 mRNA and circRNA were detected by reverse transcription-quantitative polymerase chain reaction. The values are presented as the mean \pm standard error. ${ }^{*} \mathrm{P}<0.05,{ }^{* *} \mathrm{P}<0.01,{ }^{* * *} \mathrm{P}<0.001, \mathrm{n}=3$ for each group. (C) IL-6 protein expression levels were analyzed by western blotting. GAPDH was used as an internal control. mmu, Mus musculus; circRNA, circular RNA; siRNA, small interfering RNA; NC, negative control; CGRP, calcitonin gene-related peptide; IL-6, interleukin-6.

group was significantly decreased compared with the CGRP treated group, whereas, the IL-6 mRNA expression level was increased in the CGRP + inhibitors group relative to that in the CGRP group indicating that mmu-miR-485-5p attenuated the effect of CGRP on IL-6 induction. IL-6 proteins were also evaluated (Fig. 5D). The results of western blotting were in accordance with that of RT-qPCR. Taken together, these results suggest that mmu_circRNA_007893 regulates IL-6 mRNA expression by mediating the level of mmu-miR-485-5p.

\section{Discussion}

Previous studies on CGRP and macrophages have focused on the potential pathways and kinases involved; however, the importance of circRNAs and their functions remain largely unclear. In the current study, mmu_circRNA_007893 was identified to be was involved in IL-6 induction mediated by CGRP. Furthermore, the data indicated that mmu-miR-485-5p, regulated by mmu_circRNA_007893, is a negative regulator of IL-6 expression. Overexpression of the miRNA attenuates the effect of CGRP on IL- 6 expression. Therefore, the results of the present study expand the previous understanding of the role of CGRP in macrophages and provide novel insights into the endogenous mechanism of cytokine induction.

It is reported that CGRP has an important role in inflammatory cytokine secretion mediated by $s$ in macrophage $(9,10)$. The results of the study suggest that CGRP can stimulate RAW264.7 macrophages and induce IL- 6 mRNA expression. Furthermore, CGRP acts in dose- and time-dependent manner. When the concentration of CGRP was $1 \mathrm{nM}$, the expression of IL-6 mRNA peaked, and at higher or lower
CGRP concentrations, IL-6 mRNA expression declined gradually. Similarly, IL-6 expression reached the highest level after CGRP stimulation for $2 \mathrm{~h}$, and extending or reducing the stimulation duration resulted in reduced expression of IL- 6 compared with the level at $2 \mathrm{~h}$. The results are similar to the previous finding (7). The previous study reported that CGRP can also increase the expression of IL- 6 mRNA in bone marrow-derived macrophages. However, the optimum concentration and stimulation time as not entirely consistent with the results of the present study, which may be attributes to the difference in the cell type used (7).

Recent studies have demonstrated that circRNA microarrays are a reliable and convenient method to research circRNAs and their target miRNAs and genes (25-27). Therefore, a circRNA microarray to identify the related circRNAs in the current study. Initially, the circRNAs were sorted and classified using the fold changes. Because certain studies have reported that circRNA can act as miRNA sponges to suppress the miRNA expression and thus, regulated the expression of target mRNAs $(16,20)$. Hence, it was assumed that the circRNAs that harbored more predicted miRNA binding sites had more important roles in altering the expression of their downstream miRNA (23). Subsequently, the circRNAs were sorted by the maximum number of predicted miRNA binding sites. Furthermore, a circRNA-miRNA interaction network was constructed. The miRNAs were selected according to their mirSVR scores. The network provides a pivotal reference for further investigation of the interaction of other differentially expressed circRNAs and their potential targets.

In the current study, RT-PCR, nucleic acid electrophoresis and PCR product sequencing were used to further confirm 
A

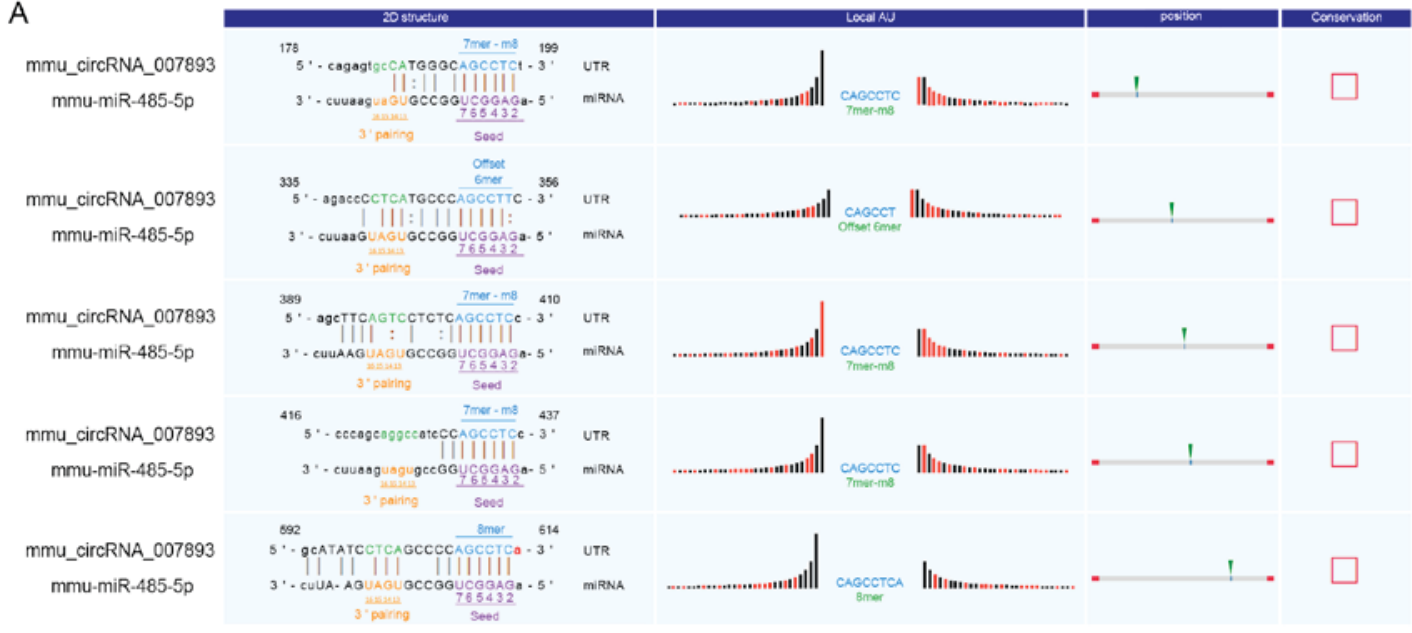

B

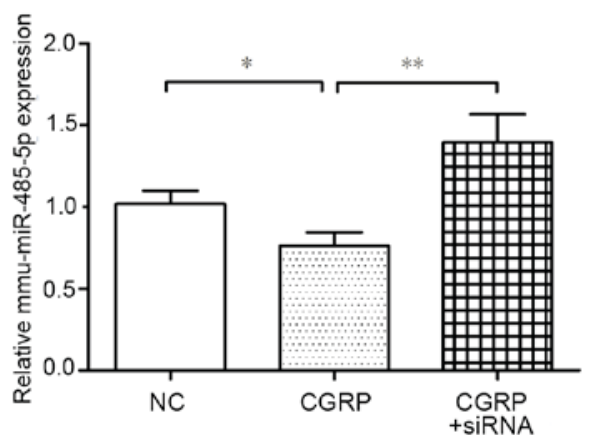

C
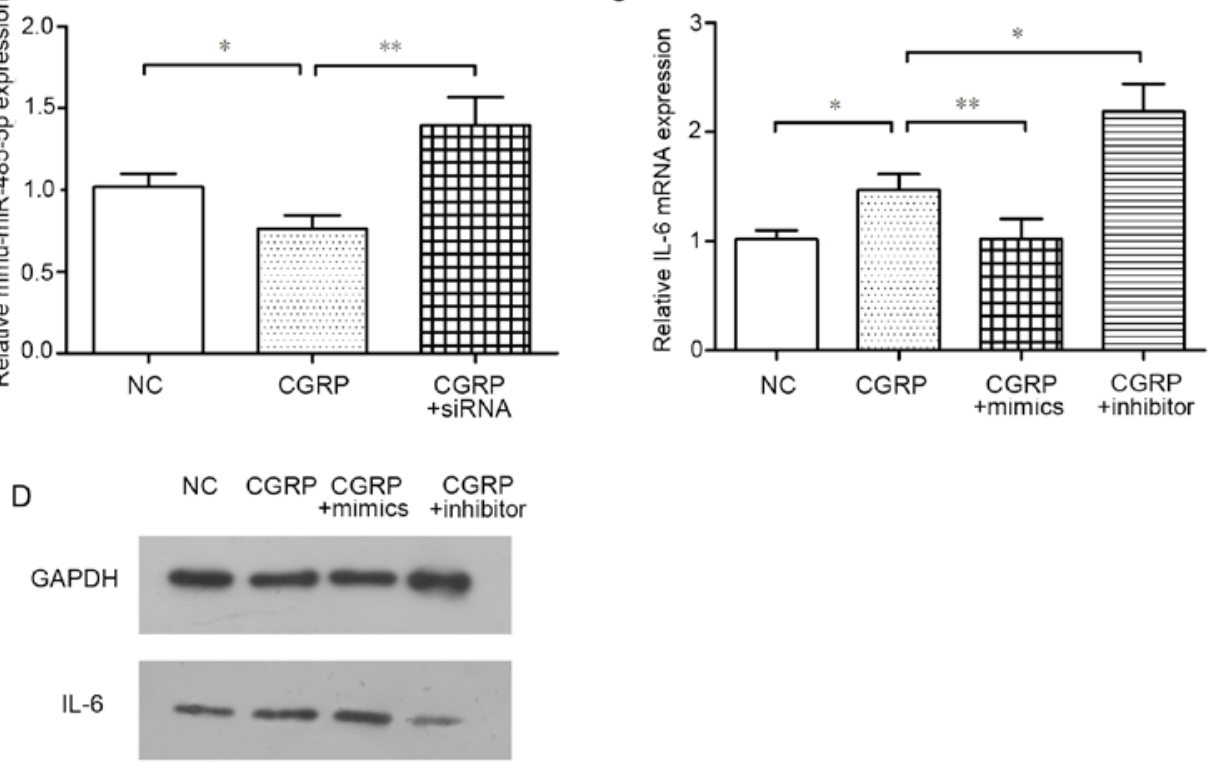

Figure 5. mmu_circRNA_007893 regulates IL-6 mRNA expression by targeting mmu-miR-485-5p. (A) mmu-miR-485-5p matched five regions in the complete sequence of mmu_circRNA_007893. (B) mmu-miR-485-5p expression in CGRP groups significantly decreased relative to that in the negative control groups $(\mathrm{P}=0.037)$. miRNA expression in CGRP + siRNA groups was significantly higher than that in CGRP groups $(\mathrm{P}=0.001)$. (C) IL-6 mRNA expression in CGRP groups was significantly higher than that in negative control groups $(\mathrm{P}=0.014)$. Expression level in $\mathrm{CGRP}+$ mimics groups decreased following overexpression of mmu-miR-485-5p using mimics, which was markedly lower than that in the CGRP groups (P=0.001). IL-6 mRNA expression in CGRP + siRNA group was significantly higher than that in the negative control group ( $\mathrm{P}=0.014)$. The IL-6 mRNA and mmu-miR-485-5p was detected by reverse transcription-quantitative polymerase chain reaction. The values are presented as the mean \pm standard error. ${ }^{*} \mathrm{P}<0.05,{ }^{* * *} \mathrm{P}<0.01, \mathrm{n}=3$ for each group. (D) IL-6 protein expression levels were analyzed by western blotting. GAPDH was used as an internal control. mmu, Mus musculus; circRNA, circular RNA; NC, negative control; CGRP, calcitonin gene-related peptide; siRNA, small interfering RNA; IL-6, interleukin-6.

the selected circRNAs. However, the data demonstrated that not all the circRNA expression levels were in accordance with the microarray analysis, and certain circRNAs were changed in the opposite direction. Similarly, this phenomenon was also reported by another study (28). This may be due to sensitive detection method of circRNA microarrays, as even minor variation or error can cause a marked difference. Thus, the results obtained by the circRNA microarray can only be a reference method and should be confirmed by further experiments.

The change of mmu_circRNA_007893 detected by RT-PCR was consistent with that of the circRNA microarray. Furthermore, interference with the expression of mmu_circRNA_007893 confirmed the association between mmu_circRNA_007893 and IL-6 mRNA. Notably, when the
mmu_circRNA_007893 expression level was knocked down using siRNA, the expression of IL-6 mRNA decreased. The result suggested that CGRP regulates IL-6 mRNA expression by affecting the level of mmu_circRNA_007893. However, other circRNAs identified in the microarray may also be involved in the IL- 6 mRNA increase and should be examined further.

A previous study reported that circRNA ciRS-7 acts as miR-7 sponge to suppress miR-7 activity, leading to increased levels of miR-7 targets (20). Several studies indicate that miR-485-5p act as a negative regulator in gastric cancer $(29,30)$. It is also reported that miR-485-5p regulates T-cell acute lymphocytic leukemia protein 1 expression in T-cell acute lymphoblastic leukemia (31). These findings demonstrated that other molecules may be mediated by 
miR-485-5p and need to be identified. Although the present study demonstrated that mmu-miR-485-5p can be regulated by mmu_circRNA_007893, the results do not exclude the involvement of other circRNAs or molecules that can regulate mmu-miR-485-5p directly or indirectly.

Overall, the results demonstrated that CGRP induces IL-6 mRNA expression, which is mediated by mmu circRNA_007893, and that mmu_circRNA_007893 functions as an endogenous miRNA sponge of mmu-miR-485-5p. Various questions should be resolved in further study. The direct association between mmu_circRNA_007893 and mmu-miR-485-5p should be confirmed. Furthermore, the effect of CGRP should be tested and detected in vivo.

\section{Acknowledgements}

This study was supported by Science \& Technology Bureau of Guangdong Province (grant no. 2013B021800278) and Research Foundation of Science \& Technology Bureau of Liwan District of Guangzhou City (grant no. 2014126055).

\section{References}

1. Amara SG, Jonas V, Rosenfeld MG, Ong ES and Evans RM: Alternative RNA processing in calcitonin gene expression generates mRNAs encoding different polypeptide products. Nature 298: 240-244, 1982

2. Li Q and Peng J: Sensory nerves and pancreatitis. Gland Surg 3: 284-292, 2014.

3. Russell FA, King R, Smillie SJ, Kodji X and Brain SD: Calcitonin gene-related peptide: Physiology and pathophysiology. Physiol Rev 94: 1099-1142, 2014.

4. Fernandes ES, Schmidhuber SM and Brain SD: Sensory-nerve-derived neuropeptides: Possible therapeutic targets. Handb Exp Pharmacol: 393-416, 2009.

5. Gasperisic R, Kovacic U, Cör A and Skaleric U: Unilateral ligature-induced periodontitis influences the expression of neuropeptides in the ipsilateral and contralateral trigeminal ganglion in rats. Arch Oral Bio 53: 659-665, 2008.

6. Baliu-Piqué M, Jusek G and Holzmann B: Neuroimmunological communication via CGRP promotes the development of a regulatory phenotype in TLR4-stimulated macrophages. Eur J Immunol 44: 3708-3716, 2014.

7. Fernandez S, Knopf MA, Bjork SK and McGillis JP: Bone marrow-derived macrophages express functional CGRP receptors and respond to CGRP by increasing transcription of c-fos and IL-6 mRNA. Cell Immunol 209: 140-148, 2001.

8. Yang W, Xv M, Yang WC, Wang N, Zhang XZ and Li WZ: Exogenous $\alpha$-calcitonin gene-related peptide attenuates lipopolysaccharide-induced acute lung injury in rats. Mol Med Rep 12: 2181-2188, 2015.

9. Liu J, Chen M and Wang X: Calcitonin gene-related peptide inhibits lipopolysaccharide-induced interleukin-12 release from mouse peritoneal macrophages, mediated by the cAMP pathway. Immunology 101: 61-67, 2000.

10. Kurashige C, Hosono K, Matsuda H, Tsujikawa K, Okamoto $\mathrm{H}$ and Majima M: Roles of receptor activity-modifying protein 1 in angiogenesis and lymphangiogenesis during skin wound healing in mice. FASEB J 28: 1237-1247, 2014.

11. Ma W, Dumont Y, Vercauteren F and Quirion R: Lipopolysaccharide induces calcitonin gene-related peptide in the RAW264.7 macrophage cell line. Immunology 130: 399-409, 2010.
12. Holzmann B: Antiinflammatory activities of CGRP modulating innate immune responses in health and disease. Curr Protein Pept Sci 14: 268-274, 2013.

13. Cocquerelle C, Mascrez B, Hétuin D and Bailleul B: Mis-splicing yields circular RNA molecules. FASEB J 7: 155-160, 1993.

14. Surono A, Takeshima Y, Wibawa T, Ikezawa M, Nonaka I and Matsuo M: Circular dystrophin RNAs consisting of exons that were skipped by alternative splicing. Hum Mol Genet 8: 493-500, 1999.

15. Jeck WR, Sorrentino JA, Wang K, Slevin MK, Burd CE, Liu J, Marzluff WF and Sharpless NE: Circular RNAs are abundant, conserved, and associated with ALU repeats. RNA 19: 141-157, 2013.

16. Meczak S, Jens M, Elefsinioti A, Torti F, Krueger J, Rybak A, Maier L, Mackowiak SD, Gregersen LH, Munschauer M, et al: Circular RNAs are a large class of animal RNAs with regulatory potency. Nature 495: 333-338, 2013.

17. Salzman J, Gawad C, Wang PL, Lacayo N and Brown PO: Circular RNAs are the predominant transcript isoform from hundreds of human genes in diverse cell types. PLoS One 7: e30733, 2012.

18. Zhang Y, Zhang XO, Chen T, Xiang JF, Yin QF, Xing YH, Zhu S, Yang L and Chen LL: Circular intronic long noncoding RNAs. Mol Cell 51: 792-806, 2013.

19. Ebbesen KK, Kjems J and Hansen TB: Circular RNAs: Identification, biogenesis and function. Biochim Biophys Acta 1859: 163-168, 2016

20. Hansen TB, Jensen TI, Clausen BH, Bramsen JB, Finsen B, Damgaard CK and Kjems J: Natural RNA circles function as efficient microRNA sponges. Nature 495: 384-388, 2013.

21. Thomas LF and Saetrom P: Circular RNAs are depleted of polymorphisms at microRNA binding site. Bioinformatics 30 : 2243-2246, 2014.

22. Westholm JO, Miura P, Olson S, Shenker S, Joseph B, Sanfilippo P, Celniker SE, Graveley BR and Lai EC: Genome-wide analysis of drosophila circular RNAs reveals their structural and sequence properties and age-dependent neural accumulation. Cell Rep 9: 1966-1980, 2014.

23. Liu Q, Zhang X, Hu X, Dai L, Fu X, Zhang J and Ao Y: Circular RNA related to the chondrocyte ECM regulates MMP13 expression by functioning as a MiR-136 'Sponge' in human cartilage degradation. Sci Rep 6: 22572, 2016.

24. Livak KJ and Schmittgen TD: Analysis of relative gene expression data using real-time quantitative PCR and the 2(-Delta Delta C(T)) method. Methods 25: 402-408, 2001.

25. Lin SP, Ye S, Long Y, Fan Y, Mao HF, Chen MT and Ma QJ: Circular RNA expression alterations are involved in OGD/R-induced neuron injury. Biochem Biophys Res Commun 471: 52-56, 2016.

26. Dou C, Cao Z, Yang B, Ding N, Hou T, Luo F, Kang F, Li J, Yang $\mathrm{X}$, Jiang $\mathrm{H}$, et al: Changing expression profiles of lncRNAs, mRNAs, circRNAs and miRNAs during osteoclastogenesis. Sci Rep 6: 21499, 2016.

27. Qu S, Song W, Yang X, Wang J, Zhang R, Zhang Z, Zhang H and $\mathrm{Li} \mathrm{H}$ : Microarray expression profile of circular RNAs in human pancreatic ductal adenocarcinoma. Genom Data 5: 385-387, 2015.

28. Wang YH, Yu XH, Luo SS and Han H: Comprehensive circular RNA profiling reveals that circular RNA100783 is involved in chronic CD28-associated CD8(+)T cell aging. Immun Aging 12: 17, 2015.

29. Jing LL and Mo XM: Reduced miR-485-5p expression predicts poor prognosis in patients with gastric cancer. Eur Rev Med Pharmacol Sci 20: 1516-1520, 2016.

30. Kang M, Ren MP, Zhao L, Li CP and Deng MM: miR-485-5p acts as a negative regulator in gastric cancer progression by targeting flotillin-1. Am J Transl Res 7: 2212-2222, 2015.

31. Correia NC, Melão A, Póvoa V, Sarmento L, Gómez de Cedrón M, Malumbres M, Enguita FJ and Barata JT: microRNAs regulate TAL1 expression in T-cell acute lymphoblastic leukemia. Oncotarget 7: 8268-8281, 2016. 